Portland State University

PDXScholar

\title{
Conditional Tests on Basins of Attraction with Finite Fields
}

Ian H. Dinwoodie

Portland State University

Follow this and additional works at: https://pdxscholar.library.pdx.edu/mth_fac

Part of the Applied Mathematics Commons, and the Mathematics Commons Let us know how access to this document benefits you.

Citation Details

Dinwoodie, I. (2014). Conditional Tests on Basins of Attraction with Finite Fields. Methodology \& Computing In Applied Probability, 16(1), 161-168. doi:10.1007/s11009-012-9304-9

This Post-Print is brought to you for free and open access. It has been accepted for inclusion in Mathematics and Statistics Faculty Publications and Presentations by an authorized administrator of PDXScholar. Please contact us if we can make this document more accessible: pdxscholar@pdx.edu. 


\title{
CONDITIONAL TESTS ON BASINS OF ATTRACTION WITH FINITE FIELDS
}

\author{
IAN H DINWOODIE \\ PORTLAND STATE UNIVERSITY
}

\begin{abstract}
An iterative method is given for computing the polynomials that vanish on the basin of attraction of a steady state in discrete polynomial dynamics with finite field coefficients. The algorithm is applied to dynamics of a T cell survival network where it is used to compare transition maps conditional on a basin of attraction.
\end{abstract}

\section{INTRODUCTION}

Complex biological networks have been modeled as discrete dynamical systems for the purposes of understanding interactions and determining steady state solutions. Logical or Boolean models, the most intuitive, have been successfully used in biology for decades ([1], [10], [13], [18], [20], [21], [22], [23]). Also, extensions to discrete states with more than two levels have been of interest, as exemplified in [8] and [12], so that on-off states may be refined to low-medium-high for example, as was used in the discretization of continuous data in [17]. Such states may be thought of as "categorical" or "ordinal" variables, but also as elements of a finite field for purposes of computation.

Many studies (see [1], [12], [16]) emphasize the importance of determining steady states and their basins of attraction - the configurations that eventually lead to the steady state. One reason is that some network configurations may exist in a mathematical model but be biologically impossible or be characteristic of rare or uninteresting mutations. Furthermore, interesting basins of attraction may be a very small fraction of the entire state space, as shown for example in [1] in a logical model of Drosophila genes. Such thin sets in a large state space can be hard to access and study.

This paper is about a method to do computations within the basin of attraction for a steady state. The method is based on representing the basin through its ideal, a set of multivariate polynomials that vanish on the basin. This representation can be very efficient, a point which we discuss in the beginning of Section 3, but in some examples it may be computationally hard and not be feasible. The results are presented for polynomials with coefficients in any finite field that serves to code the levels of each network node. The reason for using finite fields is that in certain cases the dynamics will be presented in finite field operations (see [8]), and discrete dynamics can be written this way very generally to take advantage of computational efficiencies using finite fields. The mathematical foundations are more interesting with finite fields, because there are issues of existence of roots of polynomials with coefficients in a field that is not algebraically closed that must be addressed to get a valid algorithm. However, the method can also work over fields of characteristic 0 if desired where levels can be purely categorical.

Date: April 2012, revised August 2012.

1991 Mathematics Subject Classification. Primary 13P25, 62M86.

Key words and phrases. Basin of attraction, extension theorem, polynomial dynamics, volume test. 
We illustrate on examples of six and eleven nodes in Section 3. There we will compute the conditional probability that two transition maps are identical, conditional on the basin of attraction of a steady state.

\section{Algorithm}

Consider a state space $\Omega:=\left\{\mathbf{s}=\left(s_{1}, \ldots, s_{d}\right), s_{j} \in K\right\}=K^{d}$, a $d$-fold product of any finite field $K$. The field $K$ could be $\mathcal{F}_{2}=\{0,1\}$ with operations addition modulo 2 (xor in logical notation) and multiplication like real numbers (and in logical notation). Other standard examples are $\mathcal{F}_{p}=\{0,1, \ldots, p-1\}$ with operations modulo the prime number $p$. Let $\kappa=|K|$, the number of elements in $K$.

Let $F=\left(F_{1}, \ldots, F_{d}\right)$ be a transition map or transition function or update function on $\Omega$, where $F_{j}: \Omega \rightarrow K$ and $F: \Omega \rightarrow \Omega$. This map is deterministic, it is the simplified algebraic or logical model of interactions from one time step to the next. In practice, the transition function will depend on what period of time (in seconds say) corresponds to one update or application of $F$, as longer time intervals allow for lengthier feedback and regulatory effects. The "early events $\tau=1$ " choice as in [19] precludes longer feedback mechanisms and gives greater determinism than the longer "late events $\tau=2$ " option.

A steady state $\mathbf{p}=\left(p_{1}, \ldots, p_{d}\right) \in \Omega$ has the defining property that $F(\mathbf{p})=\mathbf{p}$. Define the set of points that eventually lead to a steady state $\mathbf{p}$ :

$$
B_{\mathbf{p}}:=\cup_{k=1}^{\infty}\left\{\mathbf{s}: F^{k}(\mathbf{s})=\mathbf{p}\right\}
$$

where $F^{k}$ is the $k$-fold composition of the map $F$.

It will be convenient to use twice as many indeterminates as the number of coordinates $d$. Define the ring of polynomials $R=K\left[x_{1}, \ldots, x_{d}, y_{1}, \ldots, y_{d}\right]$. A way to study $B_{\mathbf{p}}$ is through the set of polynomials that vanish on all points in $B_{\mathbf{p}}$, that is its ideal, and the algorithm below essentially does the construction without numerically solving for preimages successively.

Define the univariate polynomial

$$
f_{K}(x)=\prod_{s \in K}(x-s) .
$$

The polynomial $f_{K}$ will be important for getting a 0 -dimensional radical ideal and extending solutions after variable elimination. The following exercise shows that $f_{K}$ is separable in common terminology.

Lemma 2.1. With $f_{K}^{\prime}$ the formal derivative of $f_{K}, \operatorname{gcd}\left(f_{K}, f_{K}^{\prime}\right)=1$.

Proof. Suppose the ged $g$ is not 1. Then one of the linear terms $x-s_{0}$ in $f_{K}$ must divide $g$, and also divide $f_{K}^{\prime}$. Hence $x-s_{0}$ must divide $\prod_{s \neq s_{0}}(x-s)=f_{K}^{\prime}-\sum_{s \neq s_{0}} \prod_{t \neq s}(x-t)$. But this is a contradiction, since $x-s_{0}$ is prime.

Define ideals

$$
\begin{aligned}
I_{K} & =\left\langle f_{K}\left(x_{1}\right), \ldots, f_{K}\left(x_{d}\right), f_{K}\left(y_{1}\right), \ldots, f_{K}\left(y_{d}\right)\right\rangle \\
F_{y x} & =\left\langle F_{1}(\mathbf{y})-x_{1}, \ldots, F_{d}(\mathbf{y})-x_{d}\right\rangle \\
F_{x y} & =\left\langle F_{1}(\mathbf{x})-y_{1}, \ldots, F_{d}(\mathbf{x})-y_{d}\right\rangle \\
I_{y p} & =\left\langle y_{1}-p_{1}, \ldots, y_{d}-p_{d}\right\rangle \\
I_{1} & =\left(F_{x y}+I_{y p}+I_{K}\right) \cap K\left[x_{1}, \ldots, x_{d}\right] .
\end{aligned}
$$


Now define recursively a sequence of ideals $I_{2}, I_{3}, I_{4}, \ldots$ by

$$
\begin{aligned}
J & =\left(F_{y x}+I_{i}+I_{K}\right) \cap K\left[y_{1}, \ldots, y_{d}\right] \\
I_{i+1} & =\left(F_{x y}+J+I_{K}\right) \cap K\left[x_{1}, \ldots, x_{d}\right], \quad i=1,2,3, \ldots
\end{aligned}
$$

Next we state a counting lemma. The dim notation refers to the dimension of the quotient ideal as a vector space over $K$.

Lemma 2.2. Consider the ideal $I_{i}^{K} \subset R$ generated by $I_{i}+I_{K}, i=1,2,3, \ldots$ Then $I_{i}^{K}$ is a radical ideal, and

$$
\operatorname{dim} R / I_{i}^{K}=\kappa^{d} \cdot\left|\left\{\mathbf{s} \in K^{d}: F^{2 i-1}(\mathbf{s})=\mathbf{p}\right\}\right| .
$$

Proof. The ideal $I_{i}^{K}$ is zero-dimensional, because it has at most $|K|^{d}$ roots in the algebraic closure of $K$. Then by Seidenberg's Lemma ([11], p.250), it is radical, since for each index $j$ both $f_{K}\left(x_{j}\right)$ and $f_{K}\left(y_{j}\right)$ belong to the ideal, and Lemma 2.1 gives the required condition on $f_{K}$. Now Theorem 3.7.9 of [11] shows that the number of solutions to polynomials in $I_{i}^{K}$ is exactly $\operatorname{dim} R / I_{i}^{K}$. It remains to show that the solutions to $I_{i}^{K}$ correspond exactly to points in $\left\{\mathbf{s} \in K^{d}: F^{2 i-1}(\mathbf{s})=\mathbf{p}\right\} \times K^{d}$. This we will prove by induction on $i$.

For $i=1$ we consider the ideal $I_{1}$. Let $\left(\mathbf{s}, \mathbf{p}^{\prime}\right) \in K^{2 d}$, for any point $\mathbf{p}^{\prime} \in K^{d}$, but with $F(\mathbf{s})=\mathbf{p}$. Then $F_{j}(\mathbf{s})-y_{j}=0, y_{j}-p_{j}=0, f_{K}\left(s_{j}\right)=0, f_{K}\left(p_{j}^{\prime}\right)=0, j=1, \ldots d$, so all polynomials in $I_{1}$ vanish at the pair $\left(\mathbf{s}, \mathbf{p}^{\prime}\right) \in K^{2 d}$. For the converse, we apply the extension theorem to the algebraic closure $\bar{K}$ of $K$. Suppose $\left(\mathbf{t}, \mathbf{p}^{\prime}\right) \in K^{2 d}$ is a solution to all polynomials in $I_{1}^{K}$. Then $\mathbf{t} \in K^{d}$ solves all equations in $I_{1}$. The extension theorem applied to the algebraic closure of $\bar{K}$ of $K([4]$, p. 25) says that $\mathbf{t}$ can be matched with the rest of a solution $\mathbf{p}^{\star} \in \bar{K}^{d}$ such that the concatenation $\left(\mathbf{t}, \mathbf{p}^{\star}\right)$ solves all equations in $F_{x y}+I_{y p}+I_{K}$. The equations in $I_{K}$ make the coordinates of $\mathbf{p}^{\star}$ lie in $K$, thus the pair $\left(\mathbf{t}, \mathbf{p}^{\star}\right) \in K^{2 d}$. Finally, the polynomials $I_{y p}$ force $\mathbf{p}^{\star}=\mathbf{p}$, so in fact $F_{j}(\mathbf{t})=p_{j}, j=1, \ldots, d$. Thus any pair $\left(\mathbf{t}, \mathbf{p}^{\prime}\right)$ that solves all polynomials in $I_{1}^{K}$ gives the point $\mathbf{t} \in K^{d}$ with $F(\mathbf{t})=\mathbf{p}$.

To continue the induction, note that the univariate polynomials that generate $I_{K}$ make extended solutions in $\bar{K}^{d}$ continue in $K^{d}$. Thus the variable elimination does not add any unwanted partial solutions, and points in $\left\{\mathbf{s}: F^{2 i-1}(\mathbf{s})=\mathbf{p}\right\}$ each correspond to $\kappa^{d}$ pairs $\left(\mathbf{s}, \mathbf{p}^{\prime}\right)$ that solve the equations in $I_{i}^{K}$.

Theorem 2.1 says to stop the iteration when $\operatorname{dim} R /\left(I_{i}+I_{K}\right)$ repeats in order to get the polynomials that vanish on the basin of attraction $B_{\mathbf{p}}$.

Theorem 2.1. Let $I_{i}^{K}=I_{i}+I_{K} \subset R, i=1,2,3, \ldots$ There exists $i^{\star}<\infty$ such that $\operatorname{dim} R / I_{i^{\star}}^{K}=$ $\operatorname{dim} R / I_{i^{\star}+1}^{K}$, and for such an integer

$$
\left|B_{\mathbf{p}}\right|=\kappa^{-d} \operatorname{dim} R / I_{i^{\star}}^{K} .
$$

Proof. By Lemma 2.2, the sequence $d_{i}:=\operatorname{dim} R / I_{i}^{K}$ is nondecreasing and counts states $\mathbf{S}$ that hit the steady state $\mathbf{p}$ at or before iteration $2 i-1$. Since the set $B_{\mathbf{p}}$ is finite, the sequence $d_{i}$ cannot increase when $i \geq \kappa^{2 d}$, so $i^{\star} \leq \kappa^{2 d}$.

Now suppose $d_{i+1}=d_{i}$. This implies that $\left\{\mathbf{s}: F^{2 i-1}(\mathbf{s})=\mathbf{p}\right\}=\left\{\mathbf{s}: F^{2 i+1}(\mathbf{s})=\mathbf{p}\right\}$, since the first is always contained in the second. If $B_{\mathbf{p}} \neq\left\{\mathbf{s}: F^{2 i-1}(\mathbf{s})=\mathbf{p}\right\}$, there would exist a point $\mathbf{s}^{\star} \in B_{\mathbf{p}}$ with $F^{2 i-1}\left(\mathbf{s}^{\star}\right) \neq \mathbf{p}$. Let $k>0$ be the first integer such that $F^{k}\left(\mathbf{s}^{\star}\right) \in$ $\left\{\mathbf{s}: F^{2 i-1}(\mathbf{s})=\mathbf{p}\right\}$. If $k=1$, then $\mathbf{s}^{\star} \in\left\{\mathbf{s}: F^{2 i+1}(\mathbf{s})=\mathbf{p}\right\} \backslash\left\{\mathbf{s}: F^{2 i-1}(\mathbf{s})=\mathbf{p}\right\}$, since $F^{2 i-1}\left(F\left(\mathbf{s}^{\star}\right)\right)=\mathbf{p}$, which contradicts $d_{i+1}=d_{i}$; if $k>1$, then $F^{k-2}\left(\mathbf{s}^{\star}\right) \in\left\{\mathbf{s}: F^{2 i+1}(\mathbf{s})=\right.$ $\mathbf{p}\} \backslash\left\{\mathbf{s}: F^{2 i-1}(\mathbf{s})=\mathbf{p}\right\}$ also contradicting $d_{i+1}=d_{i}$. Thus $B_{\mathbf{p}}$ must be $\left\{\mathbf{s}: F^{2 i-1}(\mathbf{s})=\mathbf{p}\right\}$. 


\section{Volume COMPARISONS ON BASINS OF ATTRACTION}

Here we present an application of the method of Section 2 to the comparison of two update functions. The goal is to compare two hypothetical explanations of the dynamics of a network.

Recall that the use of ideals for studying 0-dimensional varieties in statistics was developed by Riccomagno, Pistone, and Wynn [14]. Their motivation was to understand statistical models defined on an experimental design (a set of points), and the "design ideal" was useful because different term orders gave different models with identifiable parameters. For us the goal is nearly the reverse. We are seeking the "design" points - the variety that is the basin of attraction - first by computing its ideal. Then counting can be done with dimensions of quotient spaces. Note that points in $K^{d}$ can be represented as roots of a set of polynomials sometimes very efficiently. For example, the binary full factorial design in $d$ dimensions $\mathcal{D}:=\{0,1\}^{d}$ has ideal $I_{01}=\left\langle x_{1}^{2}-x_{1}, \ldots, x_{d}^{2}-x_{d}\right\rangle$ with coefficient field $F_{2}=\{0,1\}$ (in which field "-" is the same as "+"). That is, only $d$ polynomials are required to define $2^{d}$ design points. With simple transition maps on $\Omega=\mathcal{D}$ like $F_{1}(\mathbf{s})=s_{1}, F_{j}(\mathbf{s})=s_{j-1}, j=2, \ldots, d$, there are two steady states $\mathbf{0}$ and $\mathbf{1}$, and their basins of attraction can be described with one polynomial each, $\left\langle x_{1}\right\rangle$ for the basin of attraction for $\mathbf{0}$ and $\left\langle x_{1}-1\right\rangle$ for $\mathbf{1}$, in addition to the above $d$ polynomials in $I_{01}$. If $d$, which represents the number of nodes in the network, is on the order of 20 , then there are $2^{20}=1048576$ states but the basins of attraction are easily computed and can be described with just 21 polynomials. Examples like this are why the algebraic representation can be efficient. Other examples may be hard with algebra, as the worst case complexity of the Groebner basis calculations necessary for the algebraic method indicates that some examples may be impossible. Bayer and Mumford [2] discuss computational issues, with reference to work on 0-dimensional ideals that bounds the worst possible case complexity in terms of the degrees of the given polynomials $D$ and the number of coordinates $d$-the complexity may grow as a polynomial in $D^{d}$, and the degree $D$ correspond roughly to the number of "input" nodes that figure in the coordinate map so more highly coupled systems will give harder calculations.

Let $F$ and $G$ be two possible transition functions for the same network. They may come from two different studies of the literature of interaction, or from two machine learning algorithms applied to the same or different data. Our main question is how to compare them. In particular, if the data is observational data on a biological network, its states will not be arbitrary but rather related to a natural or wild-type steady state. Thus a good comparison should be conditional on the basin of attraction $B_{\mathbf{p}}$ of a steady state $\mathbf{p}$ for an accepted or null hypothesis model $F$ :

$$
q:=P\left(\{\mathbf{s}: F(\mathbf{s})=G(\mathbf{s})\} \mid B_{\mathbf{p}}\right)=E\left(I_{F=G} \mid B_{\mathbf{p}}\right)
$$

where the probability distribution $P$ on states $\mathbf{s}$ in $\Omega$ is uniform. This can be seen as an expected utility for a conditional distribution. Other distributions may also be of interest. This computation is related to the notion of volume test that began in [9] and has modern variations including the development in [6]. However, the orginal work of Hotelling was based on a rigorous development of a likelihood ratio test in a regression problem. The work of Diaconis and Efron is not founded in the same way on a likelihood ratio test, it is rather a concert of methods related to overdispersion and conditional inference that leads to a uniform distribution on constrained tables. Our interest in $q$ comes from the focus of biologists on steady states and their biological significance. 
The definition of conditional probability gives

$$
q=\frac{\left|\{F=G\} \cap B_{\mathbf{p}}\right|}{\left|B_{\mathbf{p}}\right|} .
$$

Then by Theorem 2.1 this can be computed as

$$
q=\frac{\operatorname{dim} R /\left[I_{F-G}+I_{i^{\star}}^{K}\right]}{\operatorname{dim} R / I_{i^{\star}}^{K}}
$$

where $i^{\star}$ is an index after stopping the iteration, and $I_{F-G}$ is the ideal given by

$$
I_{F-G}:=\left\langle F_{1}(\mathbf{x})-G_{1}(\mathbf{x}), F_{2}(\mathbf{x})-G_{2}(\mathbf{x}), \ldots, F_{d}(\mathbf{x})-G_{d}(\mathbf{x})\right\rangle \subset R .
$$

For comparing the maps on coordinate 1 alone, one can use just one difference $I_{F_{1}-G_{1}}=$ $\left\langle F_{1}(\mathbf{x})-G_{1}(\mathbf{x})\right\rangle$ in place of $I_{F-G}$. Many other exact conditional comparisons are possible in the same framework, the key is the set of polynomials $I_{i^{\star}}^{K}$.

Example 1. Here we consider an example from [16]. The model is logical, so we use the field $\mathcal{F}_{2}$. An update $x_{1}$ or $x_{2}$ is written as a polynomial in the form $x_{1}+x_{2}+x_{1} \cdot x_{2}$, and $x_{1}$ and $x_{2}$ is written $x_{1} \cdot x_{2}$. The polynomial defined at (1) is $f_{K}(x)=x^{2}+x$. In the table below is described a map $F$ on $d=6$ dimensions taken from Table 1 of [16].

\begin{tabular}{|l|c|c|}
\hline node & update & indeterminate \\
\hline S1P & S1P* $^{*}$ NOT (Ceramide OR Apoptosis) & $x_{1}$ \\
FLIP & FLIP* = NOT (DISC OR Apoptosis) $^{*}$ & $x_{2}$ \\
Fas & Fas* $^{*}$ NOT (S1P OR Apoptosis) & $x_{3}$ \\
Ceramide & Ceramide* = Fas AND NOT (S1P OR Apoptosis) & $x_{4}$ \\
DISC & DISC $^{*}=$ (Ceramide OR (Fas AND NOT FLIP)) AND NOT Apoptosis & $x_{5}$ \\
Apoptosis & Apoptosis* DISC OR Apoptosis & $x_{6}$ \\
\hline
\end{tabular}

This dynamic model has two steady states, a disease steady state $\mathbf{p}_{D}=110000$, and a normal steady state $\mathbf{p}_{N}=000001$. Define a second map $G$ to be the same as $F$ except on the last coordinate, where $G_{6}(\mathbf{x})=1$ consistent with the normal steady state. The algebraic computations are easily done in Singular [5], using the elimination library elim. lib for the intersection in steps (2) and (3) and vdim for computing the dimensions of the quotient ideals. The results are

$$
\begin{aligned}
& P\left(F=G \mid B_{\mathbf{p}_{N}}\right)=24 / 59=41 \% \\
& P\left(F=G \mid B_{\mathbf{p}_{D}}\right)=0 / 5=0 \%
\end{aligned}
$$

with $i^{\star}$ equal to 2 and 3 for the two basins.

Example 2. Consider an 11-node $\mathrm{T}$ cell signalling model relevant to the network of [17]. The signalling logical model described below has four steady states

$$
\begin{aligned}
& 00000000000 \\
& 11110111111 \\
& 11011111111 \\
& 11111111111 \text {. }
\end{aligned}
$$

The calculations are terminated with $i^{\star}$ at 4,2,3, and 2 iterations for the corresponding basins of attraction, and each basin counts 512 states.

The dynamics for this model are defined precisely by 


\begin{tabular}{|l|c|c|}
\hline node & logical update & polynomial \\
\hline raf $=x_{1}$ & PKA or PKC & $x_{8}+x_{9}+x_{8} x_{9}$ \\
mek $=x_{2}$ & raf or PKA or PKC & $1-\left(1-x_{1}\right)\left(1-x_{8}\right)\left(1-x_{9}\right)$ \\
plcg $=x_{3}$ & plcg & $x_{3}$ \\
PIP2 $=x_{4}$ & plcg or PIP3 & $x_{3}+x_{5}+x_{3} x_{5}$ \\
PIP3 $=x_{5}$ & PIP3 & $x_{5}$ \\
erk $=x_{6}$ & mek or PKA & $x_{2}+x_{8}+x_{2} x_{8}$ \\
akts $=x_{7}$ & PIP3 or erk or PKA & $1-\left(1-x_{8}\right)\left(1-x_{6}\right)\left(1-x_{5}\right)$ \\
PKA $=x_{8}$ & PKC & $x_{9}$ \\
PKC $=x_{9}$ & plcg or PIP2 & $x_{3}+x_{4}+x_{3} x_{4}$ \\
P38 $=x_{10}$ & PKA or PKC & $x_{8}+x_{9}+x_{8} x_{9}$ \\
JNK $=x_{11}$ & PKA or PKC & $x_{8}+x_{9}+x_{8} x_{9}$ \\
\hline
\end{tabular}

These maps are derived from the interaction diagram Figure 2 of [24], where incoming directed nodes are combined by logical disjunction, that is the or operation. Other dynamics are also compatible with known interactions in this network and the algebraic method presented here applies to all.

The experimental paper [17] remarks on the influence of PKC on PKA, reporting an unexpected influence based on Bayesian analysis of their experimental data of flow cytometry from 9 perturbations. We used tree classification [15] on their data, concatenating the nine experimental data files and discretizing to two states using a kmeans clustering method on the logarithm of responses, then using one time lag for 'autoregression' or more precisely autoclassification. The result in logical form for PKA was PKA $\vee($ plcg $\wedge$ !PKA), in polynomial from written $x_{8}+x_{3} \cdot\left(1-x_{8}\right)$. Substituting this map for coordinate 8 in the above dynamical model, a conditional comparison on the four steady states shows a value $q=524288 / 1048576=.5$ on each basin of attraction, showing significant disagreement. Also, random forests ([3]) were used to study variable importance for the tree classification method, and the variable PKC was not important in the model for predicting PKA, in fact appearing last in order of importance. Therefore we cannot see the influence of PKC on PKA in the data. However, the discretization step can be done in many ways (see [7] for a discussion of methods for biochemical networks). Other methods may be more appropriate than kmeans clustering for this application, and could also lead to somewhat different conclusions.

\section{REFERENCES}

[1] Albert, R., Othmer, H. G. (2003) The topology of the regulatory interactions predicts the expression pattern of the segment polarity genes in Drosophila melanogaster. J. Theor. Biol. 223, 1-18.

[2] Bayer, D., Mumford, D. (1993) What can be computed in algebraic geometry?. Computational algebraic geometry and commutative algebra (Cortona, 1991), Sympos. Math., XXXIV Cambridge Univ. Press, Cambridge, pp. 1-48.

[3] Breiman, L., Cutler, A. (2012). Package 'randomForest' 4.6-6. cran.r-project.org.

[4] Cox, D., Little, J., O’Shea, D. (1998) Using Algebraic Geometry. Springer, New York.

[5] Decker, W., Greuel, G.-M., Pfister, G., Schönemann, H. (2011) Singular 3-1-3 - A computer algebra system for polynomial computations. http://www.singular.uni-kl.de.

[6] Diaconis, P., and Efron, B. (1985) Testing for Independence in a Two-Way Table: New Interpretations of the Chi-Square Statistic. Ann. Statist. 13, 845-874.

[7] Dimitrova, E. S., Vera Licona, M. P., McGee, J., Laubenbacher, R. (2010) Discretization of Time Series Data. J. Comput. Biol. 17, 853-868.

[8] Hinkelmann, F., Murrugarra, D., Jarrah, A. S., Laubenbacher, R. (2001) A Mathematical Framework for Agent Based Models of Complex Biological Networks. Bull. Math. Biol. 73, 1583 - 1602.

[9] Hotelling, H. (1939) Tubes and spheres in $n$-spaces, and a class of statistical problems. Amer. J. Math. 61, 440-460. 
[10] Klamt, S., Saez-Rodriquez, J., Lindquist, J. A., Simeoni, L., Gilles, E. D. (2006) A methodology for the structural and functional analysis of signalling and regulatory networks. BMC Bioinformatics 7, 1471-2105.

[11] Kreuzer, M., Robbiano, L. (2000) Computational Commutative Algebra I. Springer, New York.

[12] Mendoza, L. (2006) A network model for the control of the differentiation process in Th cells. BioSystems 84, 101-114.

[13] Morris, M. K., Saez-Rodriguez, J., Sorger, P. K., Lauffenburger, D. A. (2010) Logic-Based Models for the Analysis of Cell Signaling Networks. Biochemistry 49, 3216-3224.

[14] Riccomagno, E., Pistone, G., Wynn, H. (2000) Algebraic Statistics. Chapman and Hall, London.

[15] Ripley, B. (2011). Package 'tree' 1.0-29. cran.r-project.org.

[16] Saadatpour, A., Wang, R-S., Liao, A., Liu, X., Loughran, T. P., Albert, I., Albert, R. (2011) Dynamical and Structural Analysis of a T Cell Survival Network Identifies Novel Candidate Therapeutic Targets for Large Granula Lymphocyte Leukemia. PLoS Comp. Biol. 7, e1002267.

[17] Sachs, K., Perez, O., Pe'er, D., Lauffenburfer, D. A., Nolan, G. P. (2005). Causal Protein-Signaling Networks Derived from Multiparameter Single-Cell Data. Science 308, 523-529.

[18] Saez-Rodriquez, J., Alexopoulos, L. G., Zhang, M., Morris, M., Lauffenburger, D. A., Sorger, P. K. (2011) Comparing Signaling Networks between Normal and Transformed Hepatocytes Using Discrete Logical Models. Cancer Res. 71, 5400-5411.

[19] Saez-Rodriguez, J., Simeoni, L., Lindquist, J. A., Hemenway, R., Bommhardt, U., Arndt, B., Haus, U.-U., Weismantel, R., Gilles, E. D., Klamt, S., Schraven, B. (2007) A logical model provides insights into T cell receptor signalling. PLos Comp. Biol. 3, 1580-1590.

[20] Schlatter, R., Schmich, K., Vizcarra, I. A., Scheurich, P., Sauter, T., Borner, C., Ederer, M., Merfort, I., Sawodny, O. (2009) ON/OFF and Beyond - A Boolean Model of Apoptosis. PLoS Comp. Biol. 5, e1000595.

[21] Stigler, B. (2006) Polynomial Dynamical Systems in Systems Biology. AMS 2006 Proceedings of Symposia in Applied Mathematics 64, 59-84.

[22] Thomas, R. (1973) Boolean formalization of genetic control circuits. J. Theor. Biol. 42, 563 - 585.

[23] Thomas, R. (1998) Laws for the dynamics of regulatory networks. Int. J. Dev. Biol. 42, 479-485.

[24] Werhli, A. V., Grzegorczyk, M., Husmeier, D. (2006) Comparative evaluation of reverse engineering gene regulatory networks with relevance networks, graphical gaussian models, and bayesian networks. Bioinformatics. 22, 2523-2531. 